\title{
Development of cell/transistor interface for real-time and noninvasive monitoring of potassium ion release based on apoptosis using biologically-coupled field effect transistor
}

\author{
Taiichiro Murakami ${ }^{1)}$, Toshiya Sakata ${ }^{1), 2)}$, Akira Matsumoto ${ }^{1), 2)}$, Madoka Takai ${ }^{1), 2),}$ \\ Kazuhiko Ishihara ${ }^{1), 2)}$, Yuji Miyahara ${ }^{1), 2), 3)}$ \\ ${ }^{1}$ Department of Materials Engineering, School of Engineering, The University of Tokyo, 7-3-1 Hongo, Bunkyo-ku, \\ Tokyo 113-8656, Japan \\ ${ }^{2}$ Center for NanoBio Integration, The University of Tokyo, 7-3-1 Hongo, Bunkyo-ku, Tokyo 113-8656, Japan \\ ${ }^{3}$ National Institute for Materials Science, 1-1 Namiki, Tsukuba-shi, Ibaraki, 305-0044, Japan \\ FAX: +81-3-5841-1842, E-mail: murakami@mpc.t.u-tokyo.ac.jp
}

We report the real-time and noninvasive detection of $\mathrm{K}^{+}$release through cell membrane using a biologically-coupled field effect transistor (bio-FET). To achieve this purpose, the cell/transistor interface, which can selectively detect $\mathrm{K}^{+}$release through the ion-channel, was developed. The $\mathrm{K}^{+}$release through the ion-channel caused by apoptosis (programmed cell death) could be electrically detected using the bio-FET with crown ether monolayer. Thus we have demonstrated the ability to analyze selectively the ion channel behavior using the bio-FET modified with the functional monolayer.

Key words: field effect transistor (FET), apoptosis, cell adhesion, ion-channel

\section{Introduction}

Recently, many types of biochips and biosensors have been developed in the fields of biological and clinical research, medical applications and drug discovery. Meanwhile, field effect transistors (FET) have been applied to biosensors; for example, enzyme-FET [1 3] and immuno-FET [4]. We have also reported some original manners for label-free and non-invasive bio-functional analyses using biologically coupled field effect transistors (bio-FET) [5,6]. The principle of bio-FET is based on the potentiometric detection of charge density changes induced at a gate insulator/solution interface accompanied by specific bio-molecular recognition events. Ion or molecular charges at the gate insulator electrostatically interact with electrons in silicon crystal across the thin gate insulator resulting in the threshold voltage change.

Particularly, we have been interested in ion transportations through membrane proteins such as ion-channels and ion-pumps at cell membrane. The platform based on the bio-FET has some great advantages in terms of real-time, noninvasive and label-free measurements, ease of downsizing and integration by the conventional semiconductor microfabrication process. Using the bio-FET, we believe that membrane protein behavior will be detected efficiently and in real-time manner for cell functional analyses on the basis of intrinsic ion or molecular charges.

Today, in the scene of drug discovery, it takes a lot of time to tailor drugs targeted for the ion-channels. The patch clump method is one of the most popular techniques often utilized to analyze ion-channels at cell membrane. However, screening of drugs based on this method is very cumbersome. The patch clump method requires great skill and a lot of experiences. This is very unlikely to provide a solid platform for high throughput analysis. On the other hand, ion-channel is expected to be one of the most important drug targets in the future. And, in order to achieve high throughput screening, it is necessary to develop a new device enabling high throughput and real-time analysis.

In this study, we have designed the biointerface between the cell membrane and the gate surface of bio-FET for sensitive and selective detection of the cell functions (Fig. 1). In particular, we have developed bio/transistor interface that can selectively detect $\mathrm{K}^{+}$ release through the ion-channel caused by apoptosis.

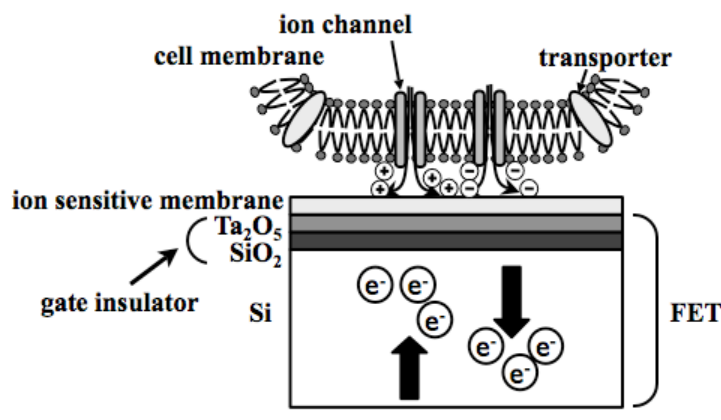

Fig. 1 Structure of bio-FET with the functional (ion sensitive) membrane 

using biologically-coupled field effect transistor

\section{Experimental}

2.1 Materials

The following chemicals and reagents were used in the experiences: carboxyethylsilanetriol sodium salt $25 \mathrm{wt} \%$ in water (Gelest), 4-aminobenzo-18-crown-6 (AB18C6, Acros Organics), 1-ethyl-3-(3-dimethyaminopropyl) carbodiimide (EDC, Dojindo), N,N-dimethylformamide (DMF, Kanto Chemical), 2[4-(2-hydroxyethyl)-1piperazinyl] ethanesulfonic acid (HEPES, Dojindo), Dulbecco's Modified Eagle Medium (DMEM, invitrogen, 11965), TNF related apoptosis inducing ligand (TRAIL, Wako). All reagent water solutions were prepared with deionized water.

\subsection{Modification of FET gate insulator surface}

We used n-channel depletion mode FET (ISFETCOM Co., Ltd.) with $\mathrm{Ta}_{2} \mathrm{O}_{5} / \mathrm{SiO}_{2}$ layer as the gate insulator. $\mathrm{Ta}_{2} \mathrm{O}_{5}$ was used as passivation layer, and it was so stable in solutions [7]. The gate width was $340 \mu \mathrm{m}$ and the length was $10 \mu \mathrm{m}$. We made functional membrane (this time, it is monolayer) sensitive to $\mathrm{K}^{+}$at the gate insulator using 18-crown-6 ether derivative (Fig. 2).

First, carboxyethylsilanetriol was used as silane coupling agent. The sensor was immersed in carboxyethylsilanetriol sodium salt $25 \mathrm{wt} \%$ in water for 24 hours, then rinsed with Milli-Q, and dried in a vacuum at $120^{\circ} \mathrm{C}$ for 1 hour. Second, $\mathrm{AB} 18 \mathrm{C} 6$ was used as ionophore of $\mathrm{K}^{+}$, and $\mathrm{EDC}$ was used as condensation agent. The sensor was immersed in AB18C6 (1mM) and EDC $(10 \mathrm{mM})$ in DMF for 24 hours and rinsed. Finally, the chemical structure like the one shown in Fig. 2 was synthesized. This monolayer doesn't contain plasticizer, and ionophore is chemically incorporated to the gate insulator, so there is no expected cytotoxicity.

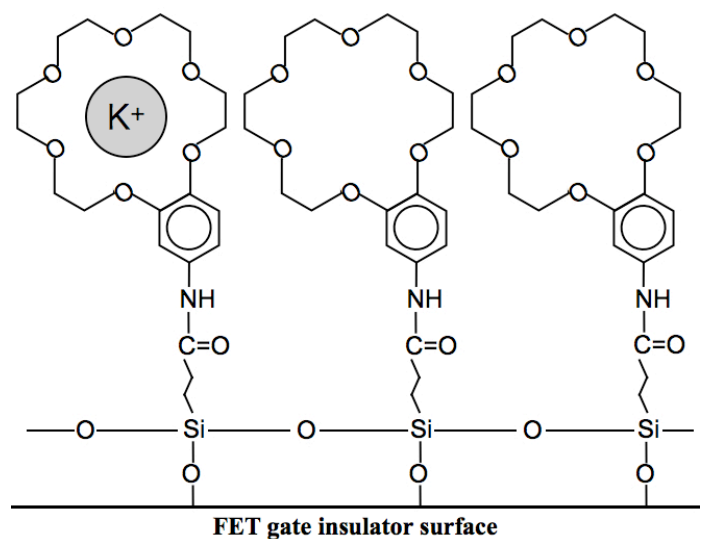

Fig. 2 Schematic representation of the functional monolayer

\subsection{Detection of variation of $\mathrm{K}^{+}$concentration}

In order to investigate the electrical characteristics of FET, a semiconductor parameter analyzer was used. Measurement was performed in HEPES buffer solution, and $\mathrm{pH}$ of the sample solution was adjusted to 7.4 with $\mathrm{Ca}(\mathrm{OH})_{2}\left(\left[\mathrm{Ca}(\mathrm{OH})_{2}\right]=1.7 \mathrm{mM}\right)$. And $\mathrm{K}^{+}$concentration was adjusted to $10^{-4} \mathrm{M}, 10^{-3} \mathrm{M}, 10^{-2} \mathrm{M}$, and $10^{-1} \mathrm{M}$ with $\mathrm{KCl}$. The prepared FET was immersed in the sample solution, and then the threshold voltage change was measured.

2.4 Measurement under cell cultural environment

To investigate the feasibility of undergoing electrical measurement under cell culturing environment, we cultured HeLa cells (derived from cervical cancer cells) at $37{ }^{\circ} \mathrm{C}$ and $5 \% \mathrm{CO}_{2}$ on the functional membrane made on the gate insulator.

Moreover, we detected $\mathrm{K}^{+}$release caused by apoptosis (for your note, earlier stage of the apoptosis, enhanced $\mathrm{K}^{+}$release takes place. $\left.[8,9]\right)$. The sensors with seeded cells were set-up in an incubator, and electrical measurement put into practice under cell culturing environment using custom-made real-time analyzer [10]. DMEM $(+1 \%$ FBS $)$ was used as culture medium. The number of seeded cells was about $10^{5}$ cells $/ \mathrm{ml}$.

The detection scheme for apoptosis is shown in Fig. 3. The prepared FET with functional monolayer was used. HeLa cells were seeded on the prepared FET, and investigation was practiced under already mentioned environment. A custom-made real-time analyzer was used in potentiometric measurement. After confirming that cells were confluent, surface potential was monitored. The apoptosis was initiated by injecting TRAIL $(500 \mathrm{ng} / \mathrm{ml})$, and the signal changes were compared to those of controls, that were free from TRAIL additions.

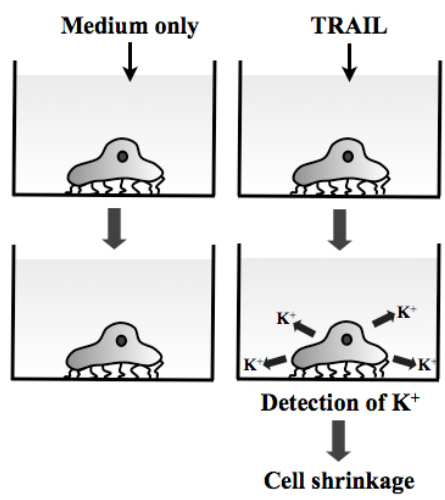

Fig. 3 Detection scheme for apoptosis

The difference between one with TRAIL and the one without TRAIL was measured.

\section{Results and Discussions}

3.1 Detection of variation of $\mathrm{K}^{+}$concentration

The effect of $\mathrm{K}^{+}$concentration on the electrical signal of prepared FET is shown in Fig. 4. This graph showed the potential difference with reference to $10^{-4} \mathrm{M}$. The electrical response of bare-FET to $\mathrm{K}^{+}$concentration was $-0.3 \mathrm{mV} /$ decade, and the electrical response of prepared FET to $\mathrm{K}^{+}$concentration was $-4.2 \mathrm{mV} /$ decade. It was relatively small compared to that of the Nernstian response. This could be because the density of ionophore immobilized onto the FET gate surface is low. Further investigation toward improved sensitivity and selectivity to $\mathrm{K}^{+}$is currently underway.

The surface potential shift to negative direction indicates the increase of $\mathrm{K}^{+}$with positive charge on the crown ether-modified gate insulator. Thus, the variation of $\mathrm{K}^{+}$concentration was successfully detected using the prepared FET. 
The charge density change at the gate insulator could be detected as a shift of potential difference of the prepared FET, so the number of crown ether per unit area related to the shift of potential difference per decade of $\mathrm{K}^{+}$concentration $(\Delta \mathrm{V})$ can be estimated [11]. $\Delta \mathrm{V}$ is expressed in Equation (1), where $\Delta \mathrm{Q}$ is charge density change per decade of $\mathrm{K}^{+}$concentration and $\mathrm{Ci}$ is gate capacitance per unit area.

$\Delta \mathrm{V}=\Delta \mathrm{Q} \times \mathrm{Ci}^{-1}$

Since $\Delta \mathrm{V}=4.2 \mathrm{mV}$ and $\mathrm{Ci}=4.3 \times 10^{-4} \mathrm{Fm}^{-2}$ for the prepared FET, the charge density change per decade is calculated to be $1.8 \times 10^{-6} \mathrm{Ccm}^{-2}$. We assume all the $\mathrm{K}^{+}$ contributed to the $\Delta \mathrm{V}$ is captured with crown ether monolayer at the solution/gate interface based on the chemical equilibrium. Considering these assumptions, the number of crown ether per unit area related to $\Delta \mathrm{V}$ is $1.1 \times 10^{9} \mathrm{~cm}^{-2}$. This is why the number of crown ether tethered per unit gate area must be more than $1.1 \times 10^{9}$ $\mathrm{cm}^{-2}$.

$\mathrm{K}^{+}$and crown ether are held in equilibrium, so the increased density of immobilized ionophore results in the increased number of $\mathrm{K}^{+}$and ionophore complex. Thus the increase of immobilization density of ionophore could give the improved sensitivity.

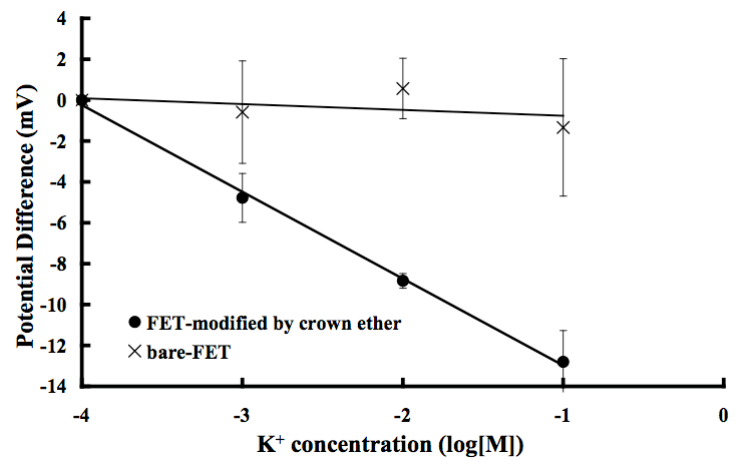

Fig. 4 Potential response for concentration of $\mathrm{K}^{+}$ using non-modified FETs and crown ether-modified FETs. The slope of potential changes is $-4.2 \mathrm{mV} /$ decade for crown ether-modified FETs.

\subsection{HeLa cells on the FET device}

The microscopic image of HeLa cells is shown in Fig. 5. This picture shows HeLa cells adhered and extended on the prepared FET. So, the microscopic observation proved clearly that HeLa cells were able to be cultured on the FET with the functional monolayer.

\subsection{Detection of $\mathrm{K}^{+}$release caused by apoptosis}

In order to clarify the reason of potential change, we developed the bio-FET modified with the crown ether that was sensitive to concentration of $\mathrm{K}^{+}$ion, and investigated the electrical signal caused by apoptosis using the functionalized bio-FET. Actually, the result showed that the surface potential of the gate surface shifted in the negative direction after introducing TRAIL (Fig. 6). This result demonstrates that $\mathrm{K}^{+}$release at early
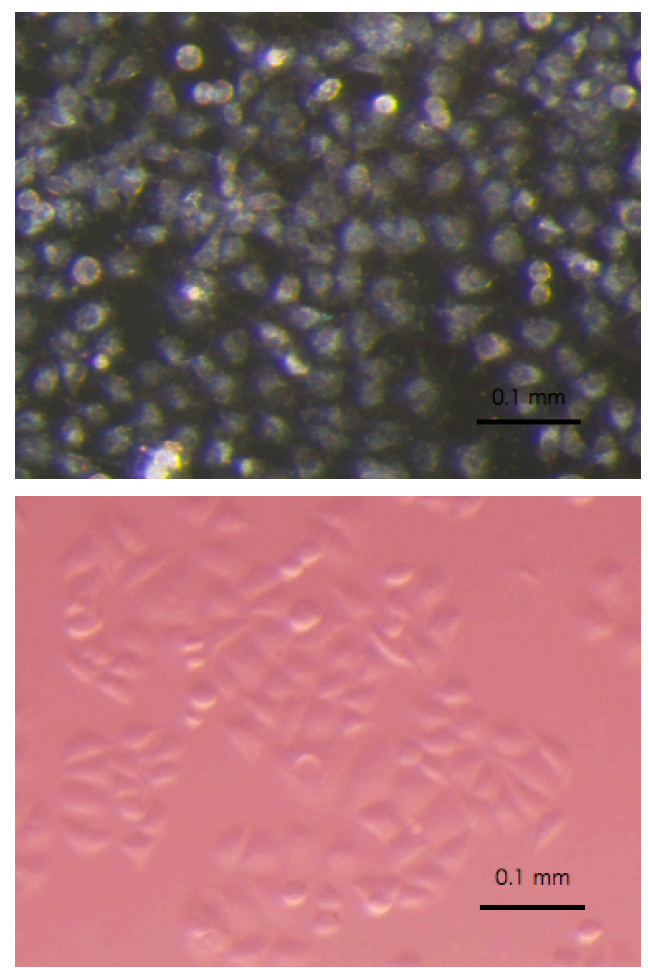

Fig. 5 Microscopic image of HeLa cells on the substrate.

Upper picture shows HeLa cells cultured on the gate insulator with functional membrane, and lower picture shows cells cultured on a normal Petri dish.

stage of apoptosis from ion channels at cell membrane was successfully detected using the bio-FET.

In this study, the differential measurements were performed using the two kinds of bio-FET; one is the bio-FET with TRAIL while another is the bio-FET without TRAIL. The differential measurement was effective to eliminate common background noises such as temperature change, non-specific adsorption, change in ion concentration and so on. The surface potential change shown in Fig. 6 was based on the differential

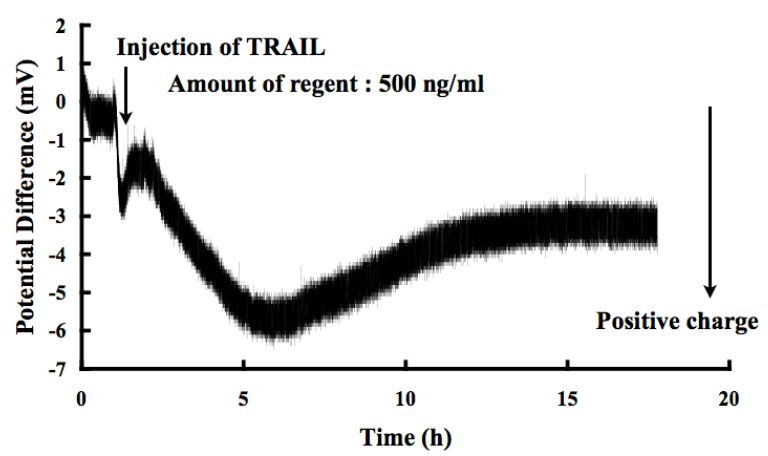

Fig. 6 Surface potential change of bio-FET for $\mathrm{K}^{+}$ release caused by apoptosis.

TRAIL was injected at $1 \mathrm{~h}$. This electrical signal was based on the differential measurements. 
measurement.

Here, we assume that $\mathrm{K}^{+}$release in the early stage of apoptosis would be trapped in the crown ether membrane at the cell/transistor interface and they would diffuse out eventually based on chemical equilibrium.

\section{Conclusions}

In conclusion, the gate-sensing surface of bio-FET could be chemically modified by $18 \mathrm{C} 6$ derivative. We could make a potassium ion sensor, on which cells could be cultured. The $\mathrm{K}^{+}$release through the ion-channel caused by apoptosis could be electrically detected using the prepared bio-FET.

We believe that we have shown the ability to analyze the ion channel behavior using the bio-FET and the platform based on the bio-FET in combination with the functional bio-interface is suitable for non-invasive, real-time and high-throughput monitoring of apoptosis for drug discovery.

\section{Acknowledgement}

We would like to thank Dr. J. Miyake and Dr. T. Kihara of Osaka University for their helpful technical advices.

\section{References}

[1] S. Caras, J. Janata: Anal. Chem. 52, 1935 (1980)

[2] Y. Miyahara, T. Moriizumi, S. Shiokawa, H Matsuoka, I. Karube, S. Suzuki: Nippon Kagaku Kaishi 6, 823 (1983)

[3] Y. Miyahara, T. Moriizumi and K. Ichimura: Sens. Actuators 7, 1 (1985)

[4] S. Collins, J. Janata: Anal. Chim. Acta 136, 93 (1982)

[5] T. Sakata, Y. Miyahara: Angew. Chem. Int. Ed. 45, 2225-2228 (2006)

[6] T. Sakata, Y. Miyahara: Anal. Chem. 80, 1493-1496 (2008)

[7] Y. Horiike, Y. Miyahara: Biochip and Biosensor edited by The polymer Science, Japan (2006)

[8] E. Maeno, Y. Ishizaki, T. Kanaseki, A. Hazama, Y. Okada: Proc. Natl. Acad. Sci. USA 17, 9487-9492 (2000)

[9] Y. Okada, E. Maeno, T. Shimizu, K. Dezaki, J. Wang, S. Morishima: J. Physio. 532.1, 3-16 (2001)

[10] T. Sakata, M. Kamahori, Y. Miyahara: Jpn. J. Appl. Phys. 44 2854-2859 (2005)

[11] T. Sakata, Y. Miyahara: Chem. BioChem. 6, 703-710 (2005)

(Received December 31, 2009; Accepted April 19, 2010) 